\title{
Index
}

Abstraction, 194

Adaptation, 84, 52, 55, 60, 63, 109, 110, 114, 115, $116,118,120,121,123,126,132,133,134,291$, 294, 298, 303, 304, 307, 309

planning, 41-43

Adaptive capacity, 298, 303, 304, 306, 309

Adaptive cycle, 293

Agriculture, 176, 190, 192, 194

Alert phase, 176

Alpine hazards, 291

Annual flow, 178, 179, 180

Anomaly/anomalies, 172, 173, 176, 180, 183, 186, 187

Aquifer responsiveness, 176

Archives, 4-5

Area in drought, 176, 183

Areal indices, 176

ARIDE, 196

ASTHyDA, 196

At-site indices, 176

Atmospheric humidity, 28, 33

Attribution, 177

Average deficit volume, 179

Average drought duration, 179, 187

Awareness, 190, 194

Bangladesh, 94

Beaufort scale, 229, 230

Bias correction, 183, 186, 187

Blueprint to safeguard Europe's water resources, 194, 196

Bonding [social capital], 306

Bounce back better, 306

Bounce: bouncing back/forward, 292, 295, 300, 308

Boundary object, 298

Bridging [social capital], 306

Business interruption costs, 258, 265

CAP, 192

Capacity, 292, 293, 294, 295, 296, 299, 300, 301, 303, $304,305,306,308,309$

Carbon cycle, 172
Catchment characteristics, 173

Catchment storage, 172

CDI, 176, 190

Change in future runoff, 187

Chile, 94

Circulation patterns, 172

Classical rainfall drought, 173

Climate change, $84,109,110,111,113,114,115$, $116,117,118,119,120,121,123,124,125,126$, $128,129,132,171,177,182,186,187$

Climate Change Act 2008, 41

Climate Change Adaptation (CCA), 101, 102-103, $105,303,304$

Climate drivers, 172, 194

Climate models, 31, 33, 34

Climate signal, 187

CMIP5, 33-34, 37, 185

$\mathrm{CO}_{2}$ fertilization, $34,39,43$

Coastal flooding, 235, 245

Coastal storms, 228, 229, 230

Cold-core cyclones, 230

Cold season, 174

Collision regime, 232, 233, 239

Colombia, 100

Combined Drought Index, 176, 190

Combined index, 176

Common Agricultural Policy, 192

Communities of circumstance, 301, 302

Communities of identity, 301, 302

Communities of interest, 301, 302

Communities of practice, 301, 302

Community, 291, 292, 293, 294, 299, 300, 301, 302, 303, 304, 305, 306, 307, 308, 309

Community resilience, 291, 292, 294, 299, 300, 305, 306, 307

Community types, 258, 260

Conceptual hydrological model, 186

Context specific, 194, 195

Contrasting climate, 173

Control period, 183, 187, 188, 189, 190

Convolution model, 238

Hydrometeorological Hazards: Interfacing Science and Policy, First Edition. Edited by Philippe Quevauviller. (C) 2015 John Wiley \& Sons, Ltd. Published 2015 by John Wiley \& Sons, Ltd. 
Coupled Model Intercomparison Project, 185

Crossan-Lane-White Organisational Learning Framework, 194

Cross-shore transport, 238, 239, 240

Cultural, 194

Cyclone, 116, 117, 119, 228, 230

Database, 63-64

Ddf 194

Decision-makers, 194

Decision support, 257, 277, 279

Deficit volume, 175, 176, 179, 186, 187

Deficits, 172

DEFRA, 39

Departure, 176

Detection, 177

DI, 187

Dialogue, 193

Dialogue Forum, 194, 195

Direct costs, 258, 261

Disaster, 52-53, 110, 114, 115, 116, 118, 120, 121 , 292, 293, 298, 299, 300, 302, 303, 304, 305, 306, 307, 308, 309

Disaster Economics, 102

Europa Reinsurance Facility, 102

Disaster Insurance

South Eastern Europe and Caucasus Catastrophe Risk Insurance Facility (SEEC CRIF), 101-102

Disaster Policy

Definition, 95

Evidence-Based Disaster Risk Policies, 101

Science-Policy Interface, 96, 101, 105

Disaster risk reduction (DRR), 95, 97-105, 292, $295,300,301,303,304$

Discharge deficit, 173

Downscaled, 183, 186

Drought, 85, 116, 117, 119, 124, 125, 126, 127, 129, $131,132,133,134,171,172,173,174,175,176$, $177,179,180,181,182,183,185,186,187,188$, $189,190,190,191,192,193,194,195,196$, 203-223

characteristics, 176,183

conditions, 172, 175, 187, 190, 191

definition, 31-32

Drought index, 32, 37

duration, 179, 187

in Europe, 31, 35-36

frequency, 172,186

generating processes, 177

hazard, 172

impact of climate change, 34, 37-39

increasing occurrence, 31,35

indicator, 183

indices, 175

intensity, 176

management, 172

occurrence, 194

projections, 186

propagation types, 173

propagation, 173, 185

severity, 187 types, 174, 177, 175, 189, 190, 191

typology, 173,177

vulnerability, 194

Drought Impact Reporter, 191

DROUGHT-R\&SPI, 193, 194, 195, 196

Dune stability factor, 236

Duration, 173, 175, 176, 179, 185, 186, 187, 192

DUROS, 238

DUROSTA, 240

Early warning, 172, 190, 191

Early Warning Systems, 93-96, 228, 242, 243

Earthquake, 291

Ecological flows, 195

Economic impact, 171

Ecosystems, 123, 124, 126, 172

EDC, 195

EDII, 192

EDO, 176, 190, 196

EmBRACE project, 291, 292, 298, 300, 301, 304, 306

Emission scenarios, 187, 189

Engineering resilience, 292, 293

Ensemble approach, 189

Ensemble member, 187, 188, 189

Environmental, 172, 182, 194

Equilibrium, 292, 293, 298

Equilibrium forcing, 228

Equilibrium models, 237, 238, 239

Equilibrium profile theory, 237, 238

Equivalent damage potential, 229

Erosion index, 237

Erosion resistance, 235, 236

Erosion response, 238

Erosion timescale, 239

European Commission, 96, 194, 195

European Drought Catalogue, 35

European Drought Centre, 195

European Drought Impact Inventory, 192, 196

European Drought Observatory, 176, 195

European Parliament, 194

European Union, 194, 195

European Water Archive, 178

Evaporation, 28, 30, 34

Evapotranspiration, 174, 177, 183

EWA, 178

Ex voto, 4

Exposure, 114, 115, 116, 118, 119, 120

Extreme events, 50, 53, 56-57, 63, 74-75

Far future, 183, 186, 187, 190

Fixed threshold, 176

Flood damage, 27

Flood Directive, 84, 129, 133

Flood/flooding, 85, 117, 119, 120, 124, 125, 126, 127, 129, 131, 132, 133, 134, 291, 292, 293, 297, 298, 299, 300, 302, 303, 304, 305, 306, 307, 308

20th Century, 32

attribution, 33

in Europe, 32

in UK, 33, 39-41

Flood management, 39-41 
Flow characteristics, 172, 183

Forcing data, 183, 196

Foredune, 237

Foreign Direct Investments (FDI), 104

Forest fires, 171

Frame of reference, 242, 243

Future climate, 33-34

Future drought, 182, 187 changes, 185

Future mean annual runoff, 187

Future scenarios, 194

Future time windows, 187

GCM, 182, 183, 187, 188, 189, 196

GCM-forced HM, 183

Gender, 293, 302

General Circulation Model, 182

Generating processes, 172, 173, 177

Geographical communities, 301, 302

Global Assessment Report on DRR, 93, 99, 103

Global carbon cycle, 172

Global climate, 172

Global drying, 183

Global hydrological models, 185

Global warming, 177, 186

Green-House Gas concentrations, 186

Green infrastructure, 194

GRI, 175

Gridded runoff, 179

Groundwater, 124, 125, 127, 130, 131, 172, 173, 175, $176,180,186,190$

abstraction, 180

characteristics, 173

drought, 173

Haiti, 94

Hazards, 172, 177, 180, 191, 192, 196, 299, 300, 303, $304,305,306,308$

Heat, 116, 118

Heat maps, 180

Heatwave, 171, 172, 291, 303, 304

Historic drought, 179, 185

Historic drought (chronology, severity), 4-12

Historic sea surges, 12-16

Historical perspective, 179

Historical studies, 181

HMs, 183, 184

Hot spot regions, 185

Human resources, 304, 306, 308

Hurricane Katrina, 228, 230

Hurricanes, 30

Hybrid Risk Model, 100

Hydroclimatological processes, 173

Hydro-climatological regions, 172

Hydrological change, 178

Hydrological characteristics, 179

Hydrological cycle, 172, 172

Hydrological drought, 172, 173, 175, 176, 181, 183, $185,186,187,188,189,190$

Hydrological drought deficits, 172

Hydrological Model, 182

Hydrometeorological data, 180, 181
Hydrometeorological extremes, 109, 110, 114, 115, $116,117,118,120,172$

Hydrometeorological fluxes, 173

Hyogo Framework for Action, 192, 245

Iceland, 94

Impact classification, 234

Impact scale, 231, 232, 233, 235, 237, 245

Impacted sectors, 174, 176, 177, 190, 191

Impacts, 172, 176, 181, 191, 192, 193, 194, 196

Impoundments, 194

Indices, 174, 175, 176, 177

Indirect costs, 259, 270

Industrial purposes, 195

Infragravity waves, 232, 241

Institutional, 194

Instrumental record, 179

Intangible costs, 259, 270

Integrated water management, 190

Interdecadal variability, 179, 181, 183

Intergovernmental Panel on Climate Change (IPCC), 32, 33, 43, 83, 109, 110, 111, 112, 113, $114,115,118,120,123,125,126,127,295,303$, 304

Intermediate future, 187

Internal climate variability, 186

International policies, 194

Inundation, 227, 232, 233, 234, 235, 245

Irrigation, 195

ISDR, 192

Italy, 104

Japan, 98-99, 104

JULES, 185

Knowledge base, 83, 84, 85, 89, 195

Lag correlations, 191

Lake, 172, 190

Land surface processes, 194

Land use, 173, 185

Large-scale climatic variability, 180

Large-scale drought, 172

Large-scale hydrological models, 179, 187, 188, 189

Learning, 63, 294, 295, 304, 306

Legal, 194

Linking [social capital], 306

Local users, 194

Long-term blend, 176

Low flow characteristics, 178

Major climates, 186

Management, 172, 173, 175, 177, 180, 182, 186, 189, $190,192,193,194$

Mandränke, 15

Manning coefficient, 237

Mediterranean, 34, 37

Memory of risk, 17-24

Meteorological drought, 172, 173, 176, 185, 186

Minor droughts, 176

Mitigate drought impacts, 194

Mitigation, 109, 110, 120, 123, 134 
Model agreement, 185

Model ensemble, 179, 185, 187, 189

Model noise, 183, 187

Model performance, 185, 189, 190

Model structure, 186

Modeling experiment, 187

Monitoring, 129, 131, 132, 172, 190, 191

Monthly flow, 178

Monthly variable threshold, 176

Morphological factor, 241

MPI-HM, 185

Multi-model ensemble, 179, 189

Multi-model ensemble mean, 179

Multi-model ensemble mean annual runoff, 179

Multi-model flow, 187

Multiple indices, 176

Multi-temporal approach, 180

N-day minimum flow, 179

National Platforms for DRR, 97, 101-105

Natural hazard, 171, 194

Natural physical system, 193

Naturalized' situation, 180

Near future, 186

Nicaragua, 99

NMDC, 176

Non-market costs, 259, 270

No-regret' actions, 41, 43

Observation, 115, 117, 118, 120

Observation-modeling framework, 180

Observed drought, 172, 177

Observed runoff, 179

OBS-forced HM, 183

One jacket size fits all, 195

Onset, 175

Organisational learning perspective, 194

Overwash, 227, 228, 231, 232, 233, 234, 235, 237, 239, 240, 241, 243, 244, 245

Overwash dune index, 237

Palmer Drought Severity Index, 176

Panarchy, 294

Pan-EU DDF, 194

Pan-European Drought Dialogue Fora, 194

Pan-European scale, 190, 191, 193, 196

Participatory framework, 50, 68, 71, 72, 73-75

Past drought, 172, 195

PDSI, 176, 177, 190

Peak-over-threshold, 231

Peat fires, 172

Penman-Monteith, 177

Performance criteria, 189

Place-based [resources], 306, 307, 308

Place-based services, 304

Policy, 172, 174, 177, 191, 192, 193, 194, 195, 196

Policy implementation, 194

Policy makers, 193, 194

Policy-making, 194, 196

Political capital/resources, 305, 306, 308

Pollution, 127, 130, 131, 132

Post-traumatic stress disorder (PTSD), 293
Potential environmental conditions, 182

Power, 298, 299, 305, 306

Precipitation, 117, 118, 119, 123, 124, 125, 127, 130, $171,172,173,174,175,176,182,183,186$

future projections, 33-34

global, 29, 33

intense rainfall, 30, 34

Precipitation anomaly, 173

Prediction, 182

Pro-active actions, 177

Pro-active measures, 181, 191

Probability density function, 174

Process-based models, 237, 239

Projected change, 187

Projection, 118, 120, 172, 177, 185, 187, 189

Propagation, 173, 176

Psycho-social

Rain-to-snow-season drought, 173

Rainfall deficit, 173

RCM, 182, 183

RCP2.6, 184, 185

RCP4.5, 184, 185

RCP8.5, 184, 185, 187, 188

RDI, 185, 187, 188

Re-analysis data, 183, 187

Recovery, 114, 120, 173, 190, 194, 293, 297, 300, 304, 308

Regional Deficiency Index, 185

Representative Concentration pathways (RCPs), 33, 37, 39, 185, 187, 188

Resilience, 58, 61-63, 73, 75, 114, 115, 116, 172, 190, 194

Resource sets, 306, 307, 308

Resources, 300, 301 303, 304, 305, 306, 307, 308

Return periods (for floods), 39

Risk, 41, 43, 109, 110, 114, 115, 116, 118, 120

assessment, 129, 130

communication, 70-72

governance, 67-70

management, 53-55, 60, 65-66, 73, 114, 116, 118 , 120

mitigation, 194, 259, 273

mitigation costs, 259, 273

reduction, 259, 273

reduction costs, 259,273

River, 172, 176, 177, 185, 189, 190, 191, 194, 196

River Basin Management Plans, 194

River basins, 189, 194

Run-up, 232, 233, 234, 235, 236, 237, 239, 240, 241, 242

Runoff

in Europe, 30, 39

high latitudes

impact of climate change, 29

Runoff deficit index, 187

SAD, 176

Saffir-Simpson Hurricane Wind Scale, 230, 231

SBEACH, 240

Science Policy Briefs, 193, 196

Science-Policy Interface, 83, 84, 86, 88, 89, 194 
Setback lines, 245

Severity, 176, 187, 188

Severity-area-duration, 176

Short-term blend, 176

Short-term trends, 180

Simulated annual runoff, 179, 187

Simulated daily runoff, 179

Simulated flow, 179

SMA, 176, 184, 185, 190

Snow cover/melt, 29, 31, 32

Snow season, 186

Social-ecological systems (SES), 292, 293, 294, 295, 303

Social learning, 304

Social resilience, 295, 299

Society, 123, 124, 125

Society of risk, 3

Socio-economic, 180, 194

Soil moisture anomalies, 184

Soil moisture depletion, 172

Soil moisture drought, 176, 186

Soil moisture replenishment, 174

Soil moisture, 172, 173, 175, 176, 180, 183, 185, 186

Soils, 173

Spatial extent, 185

Spatio-temporal evolution, 185

SPB, 193, 195

SPI, 174, 175, 176, 185, 190, 193, 194, 195, 196

SREX, 110, 114, 115, 116, 117, 118, 119, 120, 121

Stakeholders, 174, 190, 191, 192, 194

Standard operation procedures, 194

Standardized Precipitation Index, 175

State variables, 173, 176, 180

Storage processes, 186

Storm clustering, 229

Storm impact indicators, 242, 243, 244, 245

Storm impact scale, 231, 232, 233, 237, 245

Storm-induced hazards, 234

Storm severity, 229, 230, 231

Storms

20th Century, 30-31

impact of climate change, 34

Stream, 172, 176, 186

Streamflow, 173, 175, 176, 177, 178, 179, 180, 183

Subsurface storage, 176

Summer droughts, 178

Summer low flow, 179

Superstorm Sandy, 228

Sustainable livelihoods approach (SLA), 292, 305, 306, 307

Swash regime, 232, 233

Tangible costs, 259

Teleconnections, 173

Temperature, 171, 172, 173, 174, 177, 183

Terrestrial ecosystems, 172

Thailand, 104

Thames, 41

Thornthwaite, 177

Threshold, 175, 176, 180, 187

Threshold approach, 175, 176, 187

Transformability, 294, 298
Transformation, 294, 295

Trend, 172, 177, 178, 179, 180, 183

estimates, 179

in observed flow, 179

in simulated runoff, 179

studies, 176, 177, 178, 179, 180

Trend detection method, 178

Typhoons, 230

Ulrich Beck, 3

UN Int. Strategy for Disaster Reduction, 83, 85, 126, 129

Uncertainty, 30, 33, 34-35, 39, 41, 42, 43, 56-57, 71, $179,183,186,187,188,189,194,277,279$

Uncertainty analysis, 186

UNEP, 109, 112

UNESCO FRIEND-Water, 178, 196

United Nations

Global Platform for Disaster Risk Reduction, 98

Human Development Index (HDI), 93

Hyogo Framework for Action, 95, 97, 105-106

International Panel on Climate Change (IPCC), 103

United Nations Conference on Trade and Development (UNCTAD), 104

United Nations Development Programme (UNDP), 93

United Nations Framework Convention on Climate Change (UNFCCC), 102

United Nations Office for Disaster Risk Reduction (UNISDR), 96, 99, 102

The World Bank, 99, 102

United Nations Convention to Combat Desertification (UNCCD), 192

United Nations Framework Convention on Climate Change (UNFCCC), 111, 113

United Nations Office for Disaster Risk Reduction (UNISDR), 245

United States, 98

US Drought Monitor, 191

US National Drought Mitigation Centre, 176, 190

Variable threshold, 176

Vulnerability, 4, 58-60, 110, 114, 115, 116, 118, 119 , $120,121,172,190,191,193,293,298,303,304$, $305,306,307,309$

Wageningen Institute for Environment and Climate Research, 196

Warning phase, 176

WATCH, 179, 196

WATCH Forcing Dataset, 179

Watch phase, 176

Water

accounting, 195

availability, 172

consumption, 194

distribution networks, 194

management, 190, 190

managers, 174, 180, 194

metering, 194

pricing, 194 


\section{Water (Continued)}

reduction, 194

resources, 172, 173, 175, 180, 186, 189, 194, 196

re-use, 195

scarcity, 180, 181, 186, 192, 194

storage, 194

Water Framework Directive, 84, 85, 88, 124, 125, 126, 129, 130, 131, 132, 134, 192

Water Resource Management, 84, 124, 128, 129, $130,134,175,186$

WaterGAP, 185
Wave-by-wave interactions, 232

Wave group resolving models, 232

Weather type classifications, 172

Weather types, 172

Wet-to-dry-season drought, 173, 174

WFD, 190, 192, 193, 194

WIMEK-SENSE, 196

WMO, 109, 112

World Climate Research Programme, 33

XBeach, 237, 241, 243, 244

XEROCHORE, 193, 196 



